\title{
Results in astroparticle physics from the ARGO-YBJ experiment
}

\section{P. Camarri*}

on behalf of the ARGO-YBJ Collaboration

Dipartimento di Fisica, Università di Roma Tor Vergata and INFN Roma Tor Vergata

E-mail: paolo.camarrieroma2.infn.it

The ARGO-YBJ experiment, located in the Tibet region of China at 4300 meters a.s.l., has run uninterruptedly for over 5 years, namely from November 2007 till the beginning of 2013. It was designed to study astronomical gamma-ray sources in the energy range from a few hundred $\mathrm{GeV}$ up to about $100 \mathrm{TeV}$, and cosmic-ray physics in the energy range from about $1 \mathrm{TeV}$ up to few PeV. The full-coverage structure of the ARGO-YBJ detector allowed to lower the energy threshold for air-shower detection with respect to conventional sampling arrays. By using a full-coverage layer of Resistive Plate Chambers $\left(78 \times 74 \mathrm{~m}^{2}\right)$, surrounded by a guard ring, with a full area of approximately $11000 \mathrm{~m}^{2}$, the ARGO-YBJ experiment obtained important results in gammaray astronomy and cosmic-ray astrophysics: the observation of point-like gamma sources, the monitoring of AGN gamma flares, the measurement of the middle-scale cosmic-ray anisotropy and the light-component cosmic-ray spectrum are presented and discussed.

The European Physical Society Conference on High Energy Physics -EPS-HEP2013

18-24 July 2013

Stockholm, Sweden

\footnotetext{
* Speaker.
} 


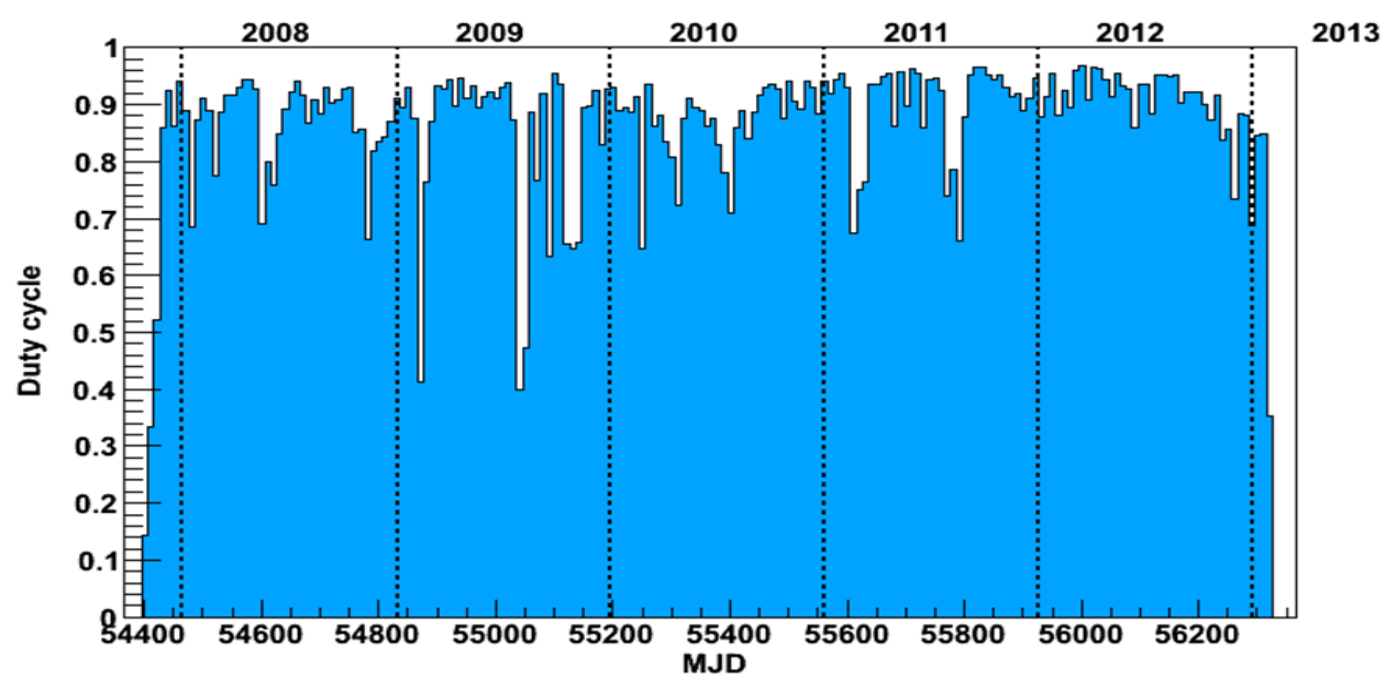

Figure 1: Time evolution of the ARGO-YBJ trigger duty cycle. The time coordinate is the Modified Julian Date (MJD).

\section{Introduction}

The ARGO-YBJ experiment [四] is a collaboration between Chinese and Italian research groups. It is located in Tibet, China, at an altitude of $4300 \mathrm{~m}$ a.s.l. in order to reconstruct air showers generated by cosmic rays with energy down to a few hundred GeV. ARGO-YBJ was designed to investigate a large number of topics in astrophysics and cosmic-ray physics: $\gamma$-ray astronomy (search for point-like sources above few hundreds of $\mathrm{GeV}$ ), search for VHE tails of $\gamma$-ray bursts above $\sim 1$ $\mathrm{GeV}$, cosmic-ray physics, Sun and heliosphere physics. The ARGO-YBJ detector is based on a full-coverage single layer of Resistive Plate Chambers (RPCs) covering a surface of $74 \times 78 \mathrm{~m}^{2}$. A group of 12 neighboring RPCs is called a "cluster", and the full-coverage part of the detector includes 130 clusters. Around this, 23 additional clusters are placed in order to obtain a better reconstruction of the shower front, which extends the experiment area to $110 \times 100 \mathrm{~m}^{2}$. The spacetime unit of the ARGO-YBJ detector is a "pad", namely a group of 8 neighboring RPC read-out strips. The single-hit time resolution is about $1.8 \mathrm{~ns}$ [[]]. Air showers are triggered by requiring a number of hits greater than 20 within a $150 \mathrm{~ns}$ time window, giving an average trigger rate of about $3.5 \mathrm{kHz}$ with a dead time of $4 \%$ and an average duty cycle greater than $86 \%$. ARGO-YBJ has been running almost uninterruptedly with its complete layout since October 2007 with a duty cycle of $90 \%$ and an average trigger rate of $3.6 \mathrm{kHz}$. The time evolution of the ARGO-YBJ trigger duty

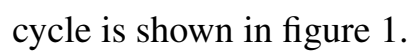

A selection of the main physics results obtained so far is presented here.

\section{Energy calibration and angular resolution in ARGO-YBJ}

An important subject of study in cosmic-ray physics is the shadowing effect exerted by the 


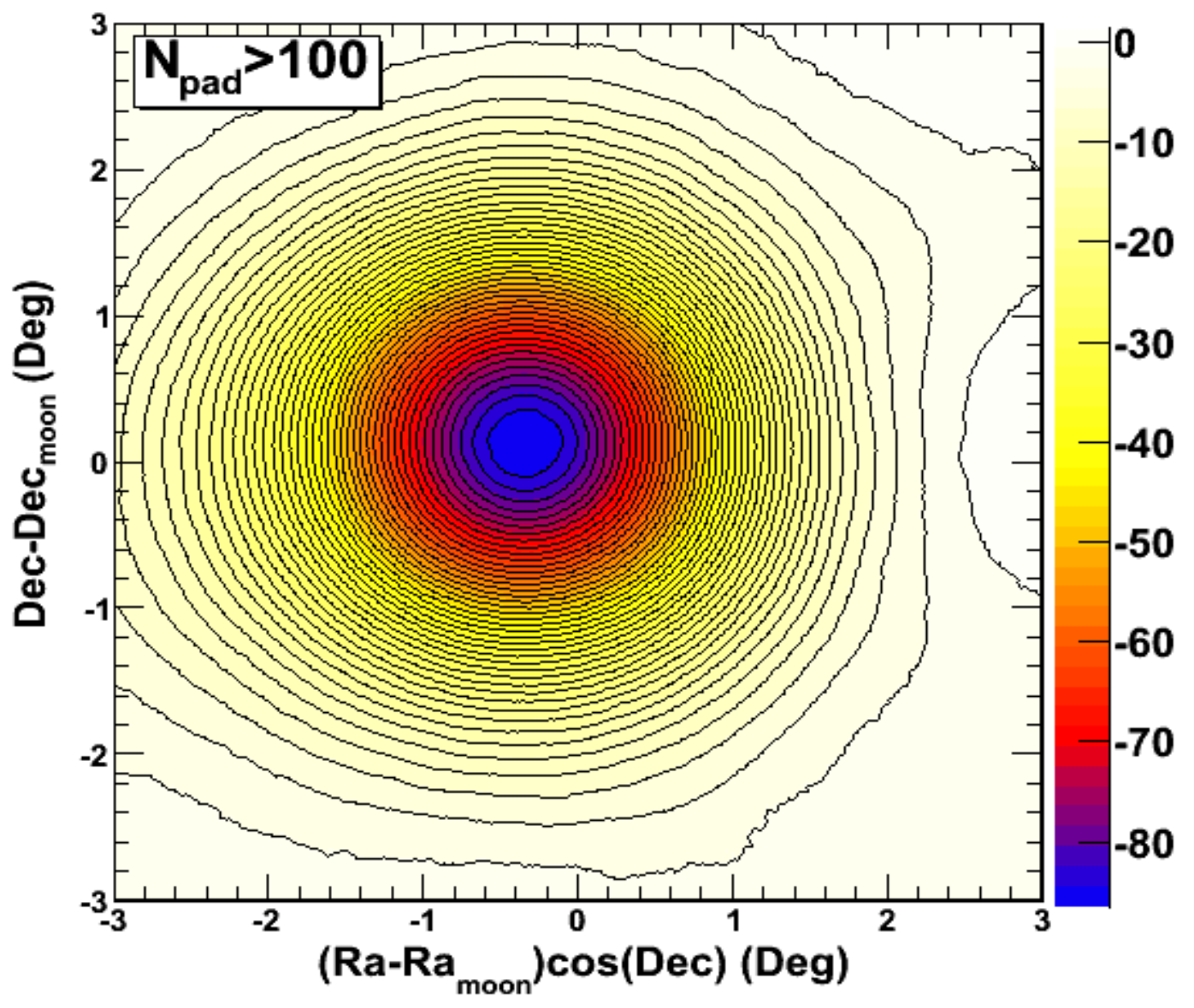

Figure 2: ARGO-YBJ sky map of the cosmic-ray flux deficit due to the Moon. This result was obtained with data collected between 2007 and 2012. The requirements for the events in this analysis were a hit multiplicity greater than 100 and a zenith angle less than $50^{\circ}$. The "Moon Shadow" was observed with a significance greater than 80 standard deviations in 5 years.

Moon on primary cosmic rays. Ground-based experiments can measure three crucial quantities related to this effect: the size, the position and the westward displacement of the deficit. From these measurements, information on the angular resolution, the pointing accuracy and the energy calibration of the detector respectively can be extracted. The angular resolution as a function of the primary energy is a crucial feature of the experiment, and this is the reason which stresses the importance of this study. Concerning the last of these three issues, the basic procedure is based on the angular bending $\Delta \theta$ of a primary cosmic ray with energy $\mathrm{E}$ and atomic number $\mathrm{Z}$ in the geomagnetic field between the Moon and the Earth $\Delta \theta \simeq 1.57^{\circ} \mathrm{Z} / E(\mathrm{TeV})$. The results coming from the data collected until the end of 2010 were described in detail in [B]]. A sky map of the cosmic-ray deficit due to the Moon measured by ARGO-YBJ is shown in Figure $\square$. 


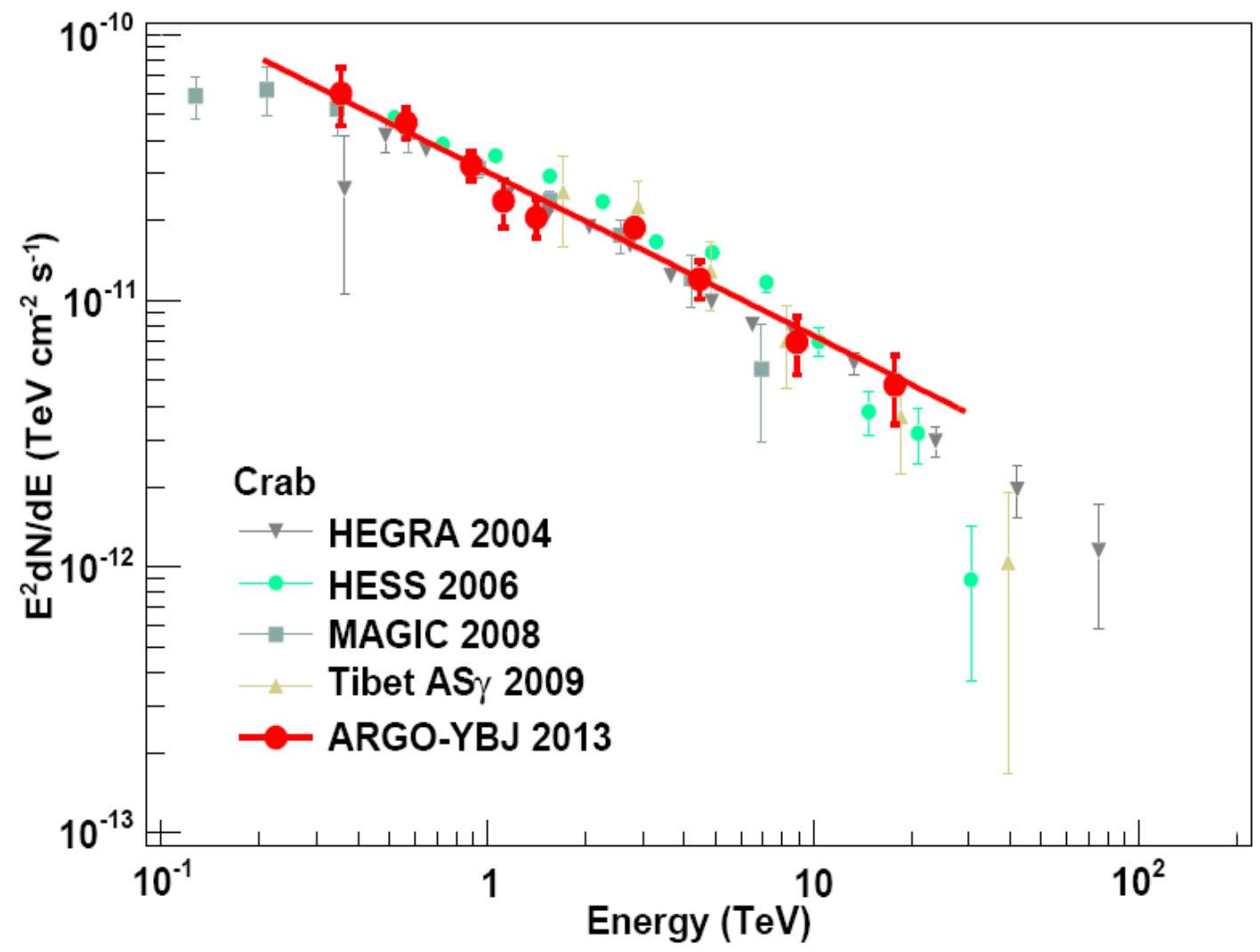

Figure 3: Comparison of the Crab $\gamma$-ray spectra measured by ARGO-YBJ (big circles and power-law fit), HESS (small circles), MAGIC (squares), HEGRA (downward triangles) and Tibet AS $\gamma$ (upward triangles).

\section{Selected results in $\gamma$-ray astronomy with ARGO-YBJ}

The analysis of the ARGO-YBJ data in $\gamma$-ray astronomy in the five years of running was mainly focused on the study of known sources, on the study of AGN flares, and on the limits on the fluence from $\gamma$-ray bursts in the "Very High Energy" range (down to a few hundred GeV). The background was evaluated by using two independent, widely established procedures: the "timeswapping" method and the "equi-zenith" method, which give equivalent results. The $\gamma$-ray emission from the Crab Nebula was detected with a significance of about 20 standard deviations in 5 years. Figure [3 shows the experimental points measured by ARGO-YBJ on the Crab Nebula energy spectrum, in perfect agreement with the known experimental results obtained by the HESS, MAGIC, HEGRA and Tibet AS $\gamma$ experiments.

A few remarkable flares from the active galactic nucleus (AGN) MRK421 were observed in the X-ray range by the ASM X-ray telescope in 2006, 2008 and 2010, and ARGO could observe $\mathrm{TeV}$ gamma emission at the same time for such flares, as shown in figure 9 for the two most recent ones.

Concerning the two flares observed on June 4-6 and June 11-13, 2008 [四], ARGO-YBJ in- 


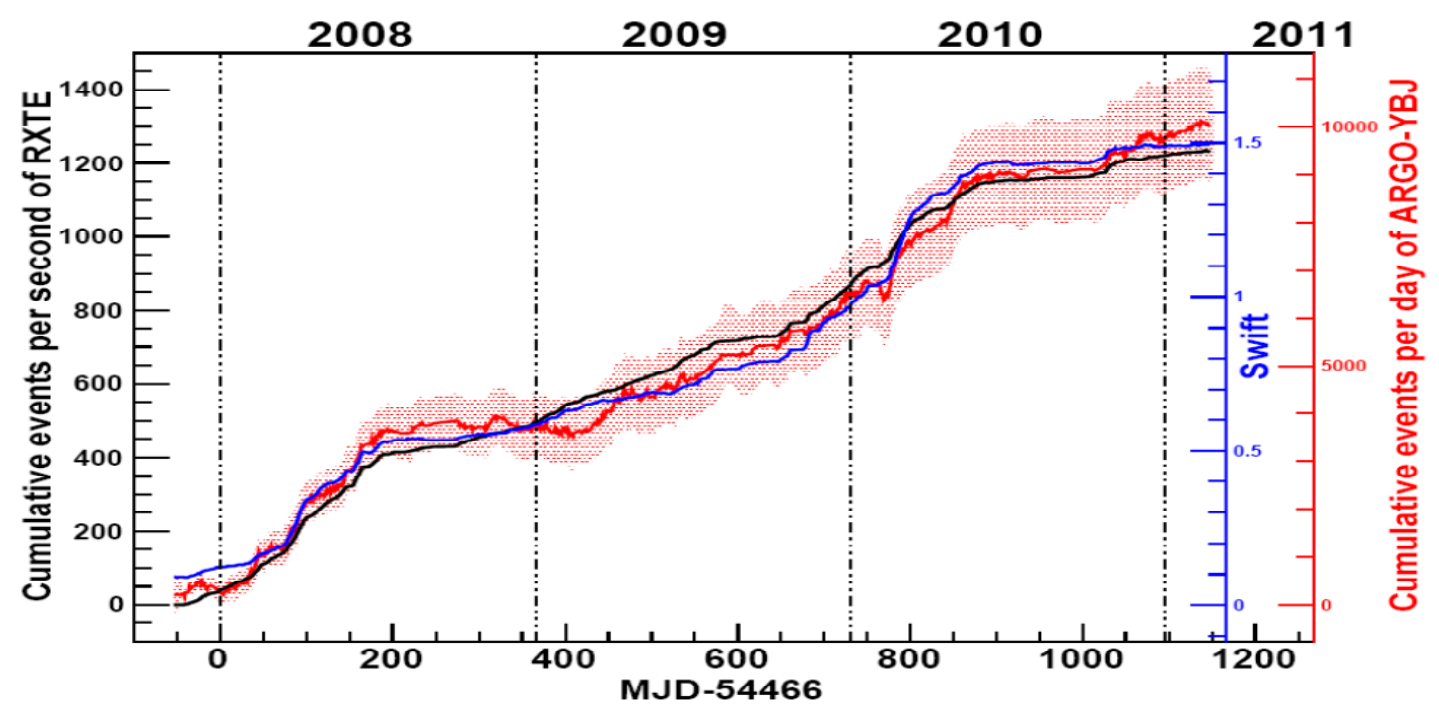

Figure 4: Comparison of the cumulative counting rates for the $\gamma$-ray emission from the Crab nebula as measured by ARGO-YBJ (red), RXTE/AMS (black) and SWIFT (blue). The flaring periods are marked with circles. The vertical coordinates have been rescaled suitably in order to obtain a direct visual comparison of the three experimental results.

vestigated the gamma emission from the same source on those days [ $[$ ]. The peak significance for the flare of June 11-13, 2008 was 4.2 standard deviations. The ARGO-YBJ data fully satisfy the relation between the spectral index and the flux resulting from the Whipple measurements of a similar flare of MRK421 [罒, suggesting that this relation is an intrinsic property of the source. One more remarkable flare from MRK421 occurred on February 2010. ARGO-YBJ observed it between February 16th and February 18th at a 6 s.d. significance level. The measured flux exceeded 3 Crab units for the duration of the observation. The importance of this measurement by ARGO-YBJ lies in the fact that for the first time a ground-based experiment could detect a flare with a 5 s.d. significance level on a daily basis.

An X-ray flare from the AGN MRK501 was detected by the RXTE/ASM telescope in October 2011 after a long "quiet" period (the previous intense X-ray flare dated back to 1997). This flare was associated to a strong emission in the TeV energy range detected by ARGO-YBJ [ [ $]$ ]. During the flaring period, the $\mathrm{TeV}$ emission was observed in 36 days with a significance greater than 6 standard deviations. In figure $\$$ the compared time behaviours for the counting rates measured by Swift/BAT (photon energy between 15 and $50 \mathrm{keV}$ ), Fermi/LAT (photon energy between $300 \mathrm{MeV}$ and a few tens of $\mathrm{GeV}$ ) and ARGO-YBJ (photon energy greater than few hundred $\mathrm{GeV}$ ) are shown; the flare detected by RXTE/ASM corresponds with the right end of the time scale, and the results from Swift/BAT and ARGO-YBJ are compatible with the above-mentioned flare.

If we consider the synchrotron self-Compton model (SSC) for the $\gamma$-ray emission from AGN, the results obtained by ARGO-YBJ strongly suggest that the model is favored during steady states while the emission spectrum is definitely harder with respect to the expectations during flares. Figure 6 shows the $\gamma$-ray energy spectrum from MRK501 as measured by several experiments: the 

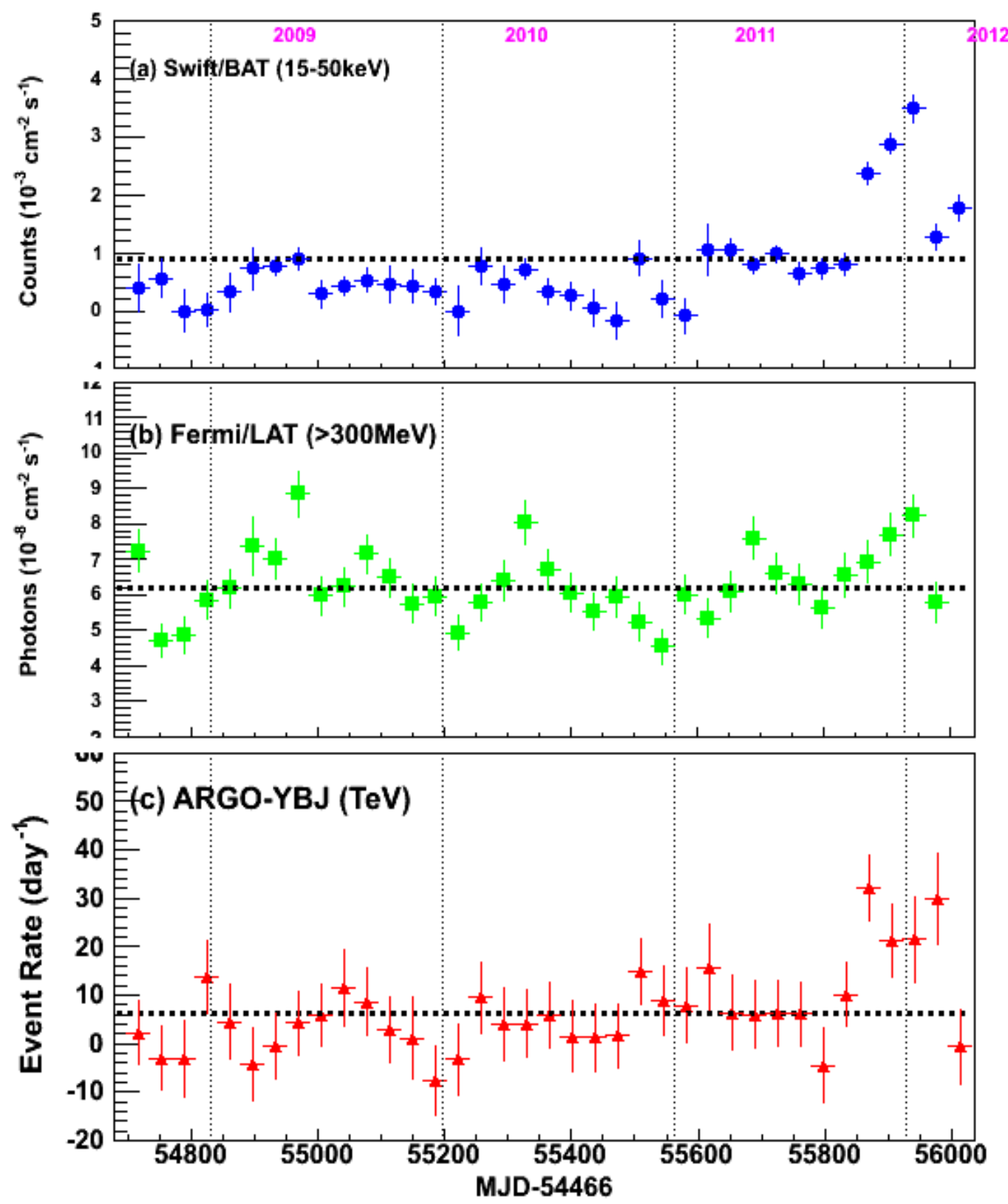

Figure 5: Comparison of the time evolution for the $\gamma$-ray counting rates emission from the AGN MRK501 as measured by Swift/BAT (a), Fermi/LAT (b) and ARGO-YBJ (c). 


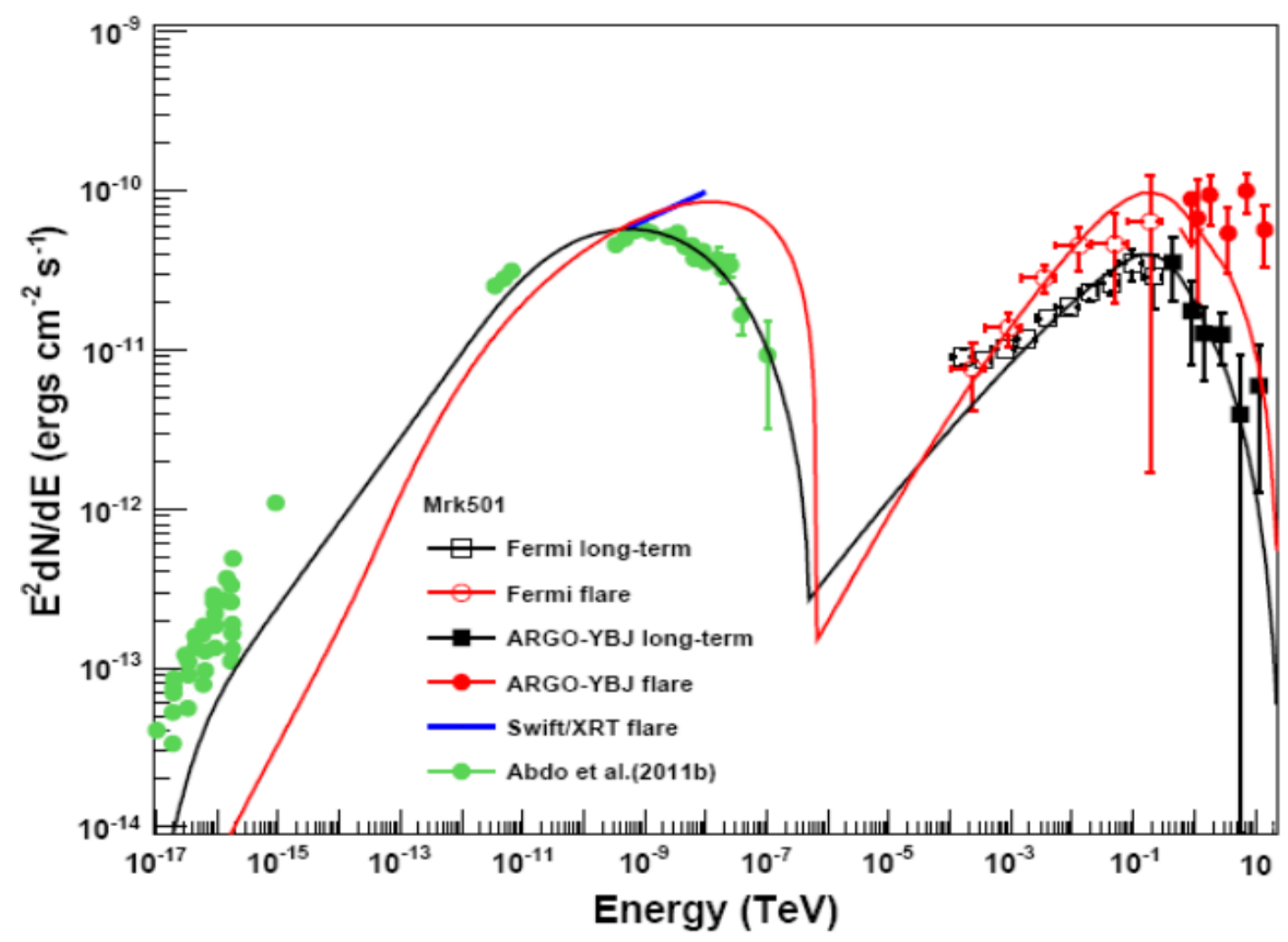

Figure 6: $\gamma$-ray spectrum of the AGN MRK501 as measured by several experiments. The high-energy measurements by ARGO-YBJ during a flare of this AGN show a clear disagreement with the expectation of the SSC model.

data from ARGO-YBJ at high energy clearly show the disagreement between the model expectation and the measurements during flares.

Finally, the sky map in declination vs. right ascension of the ARGO-YBJ sky survey for $\gamma$-ray sources is shown in figure $\mathbb{Z}$; the Crab and MRK421 are the two most significant sources measured by ARGO-YBJ at TeV energies, and are the two rightmost spots in the figure.

\section{Selected results in cosmic-ray physics with ARGO-YBJ}

The studies in cosmic-ray physics with ARGO-YBJ were focused so far on the following items: search for anisotropy at $\mathrm{TeV}$ energies, study of the moon shadow and the corresponding limit on the $\bar{p}-p$ flux ratio, proton-air interaction cross section. Concerning the medium-scale anisotropy, the ARGO-YBJ significance map of the whole visible sky with cosmic rays is shown in Fig. [ [ [8].

For this study, the data collected between 2007 and 2011 were used. A number of hit pads greater than 25 and a zenith angle $\theta<40^{\circ}$ were the requests for this analysis (corresponding to a median energy of about $1 \mathrm{TeV}$ ), and the map was obtained by using a smoothing window radius 


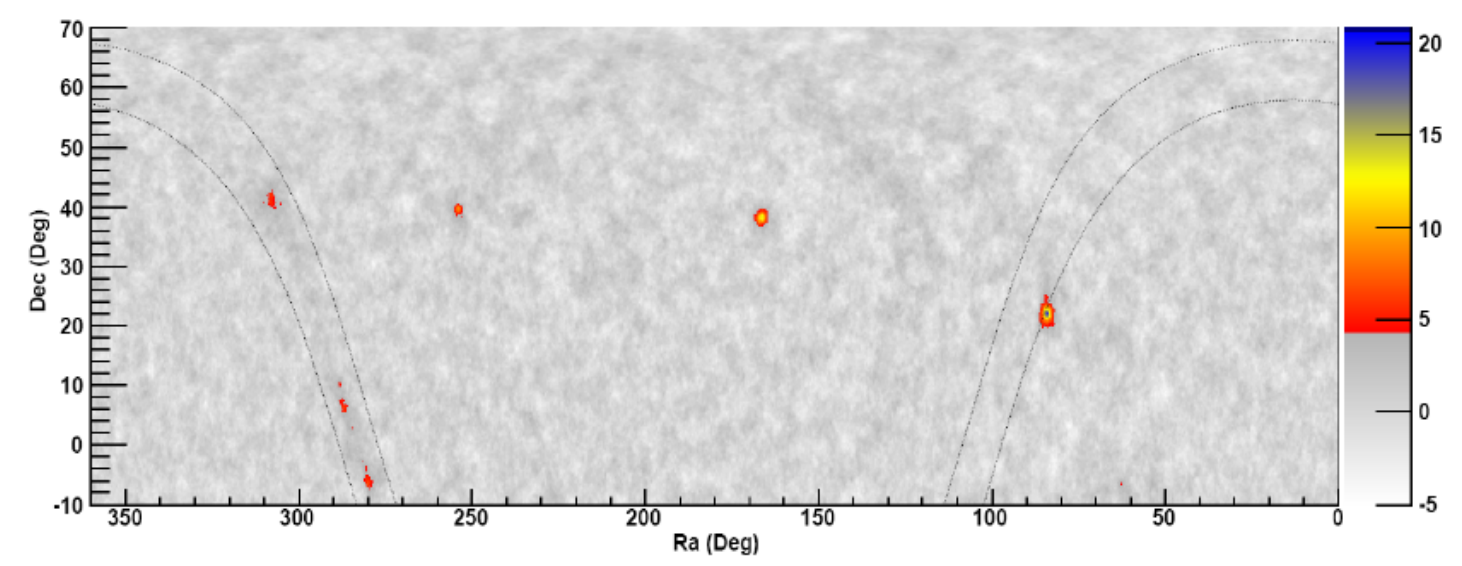

Figure 7: Sky map of the most significant high-energy $\gamma$-ray sources detected by ARGO-YBJ. The Crab and MRK421 are the first two spots from the right.
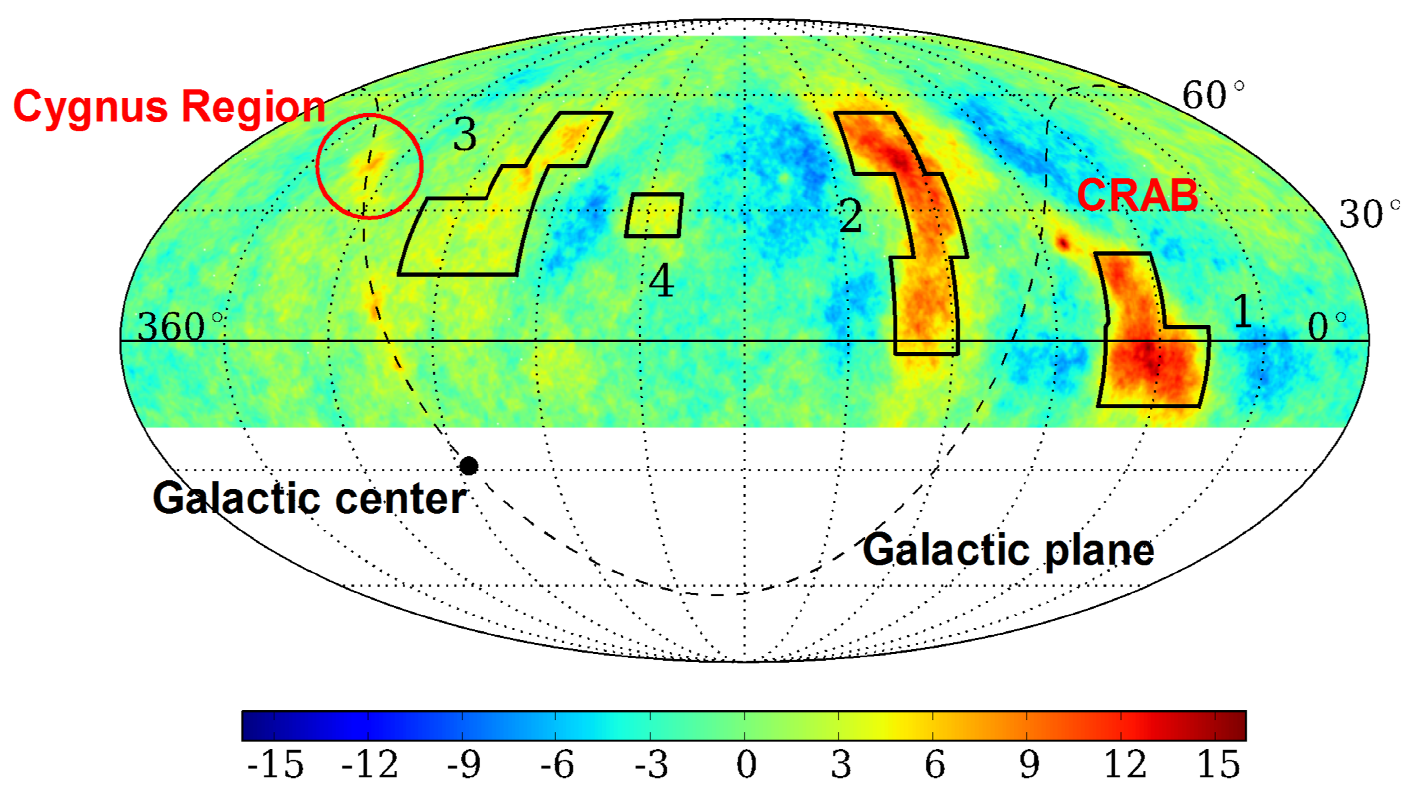

Figure 8: The ARGO-YBJ all-sky map, in equatorial coordinates. In this analysis (which uses the events with a hit multiplicity greater than 25) a smoothing was performed by using the detector Point Spread Function (PSF) for cosmic rays. The sky projection of the galactic plane is represented by the curved continuous line across the map. Four excess regions (peak significance greater than 5 s.d.) for the cosmicray flux are shown on the map. 


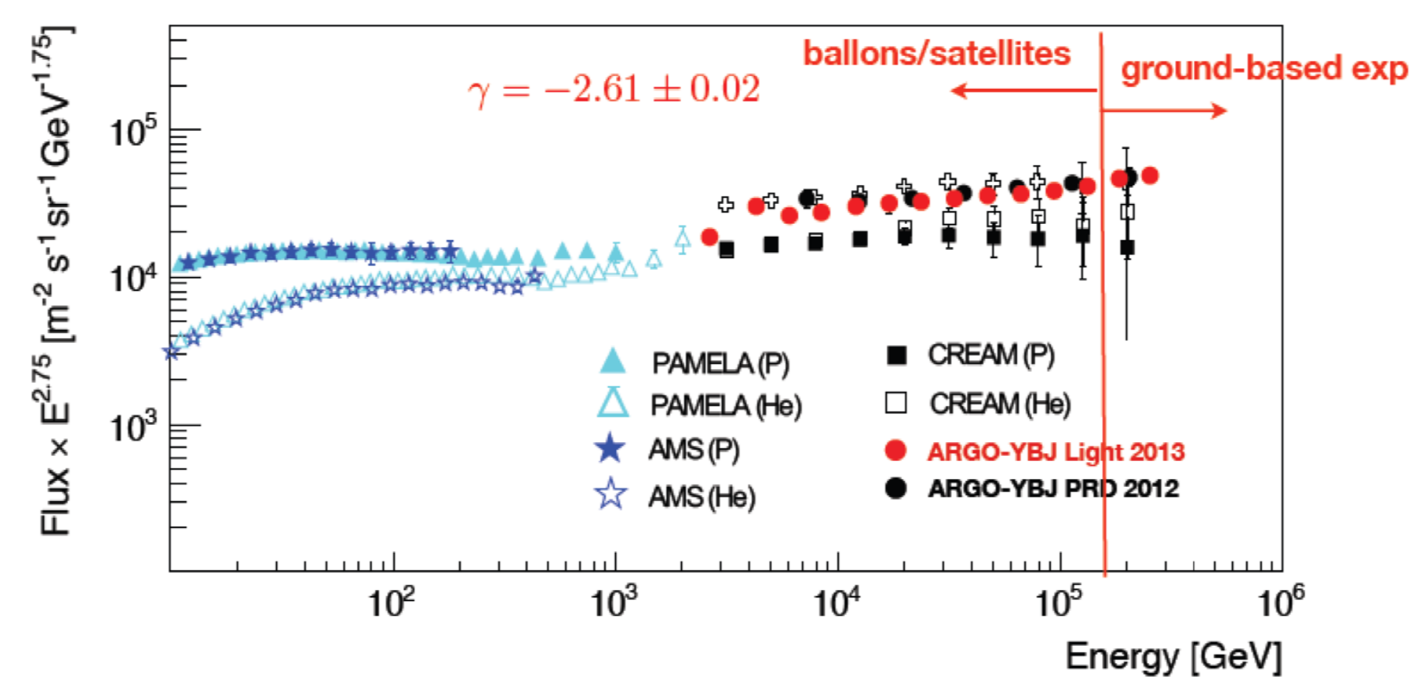

Figure 9: Light-component energy spectrum of cosmic rays in the energy range between $10 \mathrm{GeV}$ and $200 \mathrm{TeV}$, as measured by the AMS, PAMELA, CREAM and ARGO-YBJ experiments. The ARGO-YBJ results, between 5 and $200 \mathrm{TeV}$, are represented by the round points and are compatible with the CREAM measurements.

defined by the detector Point Spread Function (PSF). Four major excess regions are clearly visible. The regions 1 and 2 correspond respectively to the two excess regions of the all-sky significance map obtained by the MILAGRO experiment [Q]. Therefore, ARGO-YBJ confirms the result obtained by MILAGRO for the medium-scale anisotropy. This effect is definitely surprising, since at the $\mathrm{TeV}$ energy scale the flux of galactic cosmic rays is expected to be highly isotropic due to the scrambling in the galactic magnetic field over a very long time. A possible explanation for this puzzling experimental evidence will be the subject of future studies.

A physical result which follows directly from the Moon-shadow analysis is the upper limit on the flux ratio of antiprotons and protons in the primary cosmic radiation at the $\mathrm{TeV}$ energy scale. ARGO-YBJ set an upper limit to $5 \%$ at a median energy of $1.4 \mathrm{TeV}$ and $6 \%$ at $5 \mathrm{TeV}$ with a confidence level of $90 \%$ [प]]. These are the lowest upper limits available at the TeV scale.

A relevant results obtained by ARGO-YBJ was the measurement of the light-component energy spectrum of cosmic rays [U] in the energy range between 5 and $200 \mathrm{TeV}$, performed by using an unfolding technique based on a Bayesian approach. The ARGO-YBJ results are compatible with the measurements performed by the CREAM experiment [ए]].

\section{Conclusions}

The ARGO-YBJ experiment collected data almost uninterruptedly with its complete layout from November, 2007 till February, 2013. It obtained important results in galactic and extragalactic $\gamma$-ray astronomy, and in cosmic-ray physics as well at the energy scale from $\sim 100 \mathrm{TeV}$ down to 
a few hundred GeV. The future of the ARGO-YBJ experiment and its possible restart are being discussed at present.

\section{References}

[1] G. Aielli et al.; NIM A 562 (2006) 92-96.

[2] G. Aielli et al.; NIM A 608 (2009) 246-250.

[3] B. Bartoli et al. (the ARGO-YBJ collaboration); Phys. Rev. D 84 (2011) 022003.

[4] I. Donnarumma et al.; ApJ 691 (2009) L13-L19.

[5] G. Aielli et al. (the ARGO-YBJ collaboration); ApJ Letters 714 (2010) L208-L212.

[6] F. Krennrich et al. (the WHIPPLE collaboration); ApJ 575 (2002) L9-L13.

[7] B. Bartoli et al. (the ARGO-YBJ collaboration); ApJ 758 (2012) 2.

[8] R. Iuppa; Ph.D. thesis (University of Roma "Tor Vergata”, 2012). G. Di Sciascio on behalf of the ARGO-YBJ collaboration; arXiv: 1010.4401.

[9] A. A. Abdo et al. (the MILAGRO collaboration); Phys. Rev. Lett. 101 (2008) 221101.

[10] B. Bartoli et al. (the ARGO-YBJ collaboration); Phys. Rev. D 85 (2012) 022002.

[11] B. Bartoli et al. (the ARGO-YBJ collaboration); Phys. Rev. D 85 (2012) 092005.

[12] H. S. Ahn et al., ApJ 715 (2010) 1400. 